\title{
A Novel Reaching Law for Sliding Mode Control of Uncertain Discrete-Time Systems
}

\author{
Qian Xu $\mathbb{D}^{1},{ }^{1}$ H. P. Du $\mathbb{D}^{2},{ }^{2}$ B. He, ${ }^{3}$ T. H. Yan $\mathbb{D}^{1},{ }^{1}$ W. H. Li $\mathbb{D},{ }^{2}$ and S. S. Sun ${ }^{2}$ \\ ${ }^{1}$ School of Mechanical \& Electrical Engineering, China Jiliang University, China \\ ${ }^{2}$ Faculty of Engineering \& Information System, University of Wollongong, Australia \\ ${ }^{3}$ School of Information Science \& Engineering, Ocean University of China, China \\ Correspondence should be addressed to T. H. Yan; thyan@163.com
}

Received 8 May 2018; Revised 10 July 2018; Accepted 31 July 2018; Published 15 August 2018

Academic Editor: Andrzej Bartoszewicz

Copyright (C) 2018 Qian Xu et al. This is an open access article distributed under the Creative Commons Attribution License, which permits unrestricted use, distribution, and reproduction in any medium, provided the original work is properly cited.

\begin{abstract}
This paper proposed a new sliding mode control algorithm for discrete-time systems with matched uncertainty. The new control algorithm is characterized by a new discrete switching surface. Although the exponential reaching law can reduce oscillation, the control effectiveness will be suppressed when the rate of change of disturbance is high. The exponential reaching law cannot force the system states to approach sliding surface $s(k)=0$. In order to solve the contradiction between guaranteeing the basic property of quasi-sliding mode and reducing oscillation, a new discrete reaching law is proposed to improve the reaching process of discrete exponent reaching laws. The proposed method not only can force system state to approach the sliding surface $s(k)=0$ in less width of the switching manifold than existing studies, but also can alleviate chattering when the system representative points are near zero point. Simulation results are provided to validate the feasibility and reasonability of the method.
\end{abstract}

\section{Introduction}

Continuous-time variable-structure control theory and its application have been extensively studied since the early 1960s [1-3]. Thanks to their computational efficiency and robustness $[4,5]$, this control method has been applied widely and relevant results can be found in [6-9]. It works by applying a switching controller to bring the state of the system to a predefined sliding surface in finite time. Certain desired properties such as prescribed decay speed, good transient performance, and, especially, complete insensitivity to matched uncertainties can be obtained.

Since the advent of digital computation, most continuous systems are treated in their discretised forms. Thus, research on the discrete-time sliding mode control (DSMC) is increasing [10-13]. A discrete-time controller drives the system representative point into a predefined hyperplane (sliding surface), once the sliding surface is reached, the system under the controller is invariant against parameter uncertainties and external disturbance [14-17]. A large quantity of DSMC methodologies have been established [2, 18-21]. In order to achieve that objective, two different approaches can be taken. One is to assume a certain control algorithm and demonstrate that this algorithm ensures stability of the sliding motion on the hyperplane; another approach is to apply the reaching law approach. The reaching law approach was first proposed by Gao and Hung [22], based on the equality type of reaching conditions [23-27]. The control of discrete-time systems via using the reaching law approach has been used in many significant studies [28-30]. Gao et al. [23] further developed a discrete reaching law, in which a notion of quasi-sliding mode (QSM) is developed, the QSM controller will drive state trajectory into the switching manifold in finite steps and then undergoes a zigzag motion within the switching manifold. The manifold around the sliding surface is called the band of quasi-sliding domain, expressed by $\Delta(\Delta>0)$. Bartoszewicz et al. proposed a type of nonswitching reaching law in $[26,31]$ based on the idea that the system states are not required to cross the sliding surface in each successive step. Qu et al. [32] proposed a dynamic discrete-time SMC for uncertain systems with a disturbance compensator to alleviate chattering. In [33], Niu et al. proposed a novel reaching law for systems with 
external disturbances. In order to reduce the chattering which is caused by the sign function, many researchers employed a continuous term instead of the sign function [34]. Since the sign function is replaced, the advantage of the QSM, i.e., robustness, is reduced at the same time.

The QSM has an obvious advantage such as alleviation of chattering when the system state trajectory approaches the sliding surface. Generally, the trajectory of DSMC system cannot reach the origin point but chattering surrounds zero point [26]. A primary reason is that the switching frequency of the controller is not infinite. Although infinite-frequency switching is theoretically feasible from the robustness point of view, it is usually hard to achieve in practical systems because of physical constraints. In order to solve the inherent shortcoming of DSMC systems, many scholars proposed novel reaching laws; for example, Andrzej Bartoszewicz proposed some novel reaching laws $[26,31]$ which are different from Gao et al. [23]. These reaching laws are based on the idea that the system state is not required to cross the sliding surface in each successive control step, so they can alleviate undesired chattering; but for the nonswitching type reaching law, some advantages of QSM are also lost at the same time, such as the robustness of system. Besides the above approaches of alleviating undesirable chattering, many other approaches have been proposed. More precisely, a strategy combining the reaching law and fast-output-sampling method with output feedback is proposed in [28]. Chakrabary et al. [3] introduced a generalized discrete-time reaching law, which contains functions not only in the sliding state, but also in other known states. Eun et al. [35] present a disturbance compensator into the reaching law approach to improve the close-loop system control performance, which estimated the disturbance within certain estimation errors. A fuzzy sliding mode control for discrete-time system is proposed in [36]. To suppress chattering, the continuous function is employed instead of the signum function [37]. The difference function is also adopted to further narrow the width of the QSMD [38]. A chattering-free digital SMC with state observer and disturbance rejection is presented in [39-41]. In previous works, assuming the change rate of uncertainty is slow, designing a reaching law can obtain good effectiveness of chattering-free digital SMC $[20,42]$, but that rarely occurs in practical systems.

In order to solve the aforementioned problems, this paper proposed a novel reaching law which contains a piece-wise function term $w(\Delta, x)$. The piece-wise function contains two parts, one of them is $w(\Delta, s(k))=\operatorname{sign}(s(k))$, when $|s(k)| \geq \Delta$; another one is $w(\Delta, s(k))=\tan (\pi s(k) / 4 \Delta)$, when $|s(k)|<\Delta$, where $\Delta$ is the width of quasi-sliding mode band. The proposed reaching law retains the switching function of the signum function; i.e., it can force the system state trajectories across the sliding surface in each successive step, which ensures the robustness of controlled system. Thus, a new sliding mode controller is further developed based on the proposed reaching law. One unique feature of the proposed controller lies in that the proposed reaching law can alleviate chattering greatly, especially when the rate of change of disturbance is high, and the representative point can more quickly reach the sliding surface from any initial point at the same time. From the above introduction, the proposed reaching law may apply in the hard disk drives control system and high-speed $x-y$ servo platform.

The remainder of this paper is organized as follows: In Section 2, the notations and the most important definitions used throughout the paper are presented. The novel discretetime reaching law is designed in Section 3, the reaching steps and systems dynamics of the proposed method are also analysed in this section. In Section 4, the control strategy is modified, so that makes it applicable to perturbed systems; meanwhile, the properties of the modified reaching law are analysed, and the convergence property is proven. In Section 5, the effectiveness of the proposed method is validated by one numerical example.

\section{Definition and Problem Formation}

The general description of a single-input control system is as follows:

$$
\dot{x}=A x(t)+B(u(t)+f(x, t))
$$

where $x(t) \in R^{n}$ is the system state, $u(t) \in R^{1}$ is the control input, and $f(x, t)$ denotes the matched uncertainty or external disturbance. $A, B$ are known real constant matrices of appropriate dimensions. Without loss of generally, it is assumed that the pair $(A, B)$ is completely controllable, and the uncertainty or disturbance $f(x, t)$ is bounded with a known constant bound.

$$
|f(x, t)| \leq D
$$

where $D$ is a positive constant.

By applying the zero-order-holder $(\mathrm{ZOH})$ sampling [43], i.e., $u(t)=u(k)$, over the time interval $[k T,(k+1) T]$, where $T$ is the sampling period, the continuous-time system (1) under the discrete-time control law $u(k)$ can be written in a discrete form as follows:

$$
x(k+1)=G x(k)+\Gamma(u(k)+d(k))
$$

where $x(k)=x(k T), u(k)=u(k T), G=e^{A T}, \Gamma=\int_{0}^{T} e^{A \tau} d \tau B$, and $d(k)=\int_{0}^{T} e^{A \tau} B f((k+1)-\tau) d \tau$.

The objective of this paper is to design a novel DSMC algorithm which ensures that system (3) has the following properties: for any initial state $x(0)$, the system state trajectory will monotonically approach the switching hyperplane:

$$
s(k)=C^{T} x(k)=\sum_{i=1}^{n} c_{i} x_{i}(k)=0
$$

where $C=\left[c_{1}, c_{2} \cdots c_{n}\right]$ is designed such that $C^{T} \Gamma \neq 0$, and then sliding variable $s(k)$ will drive the system representative points onto the zero point when traveling along the switching surface:

$$
s=\left\{x(k) \mid C^{T} x(k)=0\right\}
$$


Definition 1. The matched uncertainty of the discrete-time system is bounded so that the following relation holds:

$$
\begin{aligned}
& d_{l} \leq d_{s(k)}=C^{T} \Gamma d(k) \leq d_{m} \\
& d_{1}=\frac{d_{l}+d_{m}}{2} \\
& d_{2}=\frac{d_{m}-d_{l}}{2}
\end{aligned}
$$

where $d_{l}$ is the minimum value of $d_{s}(k)$, and $d_{m}$ is the maximum value of $d_{s}(k)$.

Definition 2. A DSMC system can be called a quasi-sliding mode control (QSMC) system if the zigzag motion stays within $\Delta$-neighbourhood of the designed switching surface; i.e., $|s(k)| \leq \Delta$ for $k \geq k^{*}$, where $\Delta$ is the width of the quasisliding domain and $k^{*}$ is a positive constant.

Remark 3. This definition of quasi-sliding mode is essentially different from the one proposed by Gao [23], because the continuous term $w[\Delta, s(k)]$ is employed instead of the signum function. The proposed reaching law has two merits, one of them is that it can adapt to the variation of the variable $s(k)$; another is that it can ensure that the state trajectory has smaller vibration around predefined sliding surface.

Definition 4. The discrete-time system (3) satisfies the reaching condition of QSM in $\Delta$-neighbourhood of a specified switching surface if, for any $k>0$, the following conditions are satisfied:

$$
\begin{gathered}
s(k)>\Delta \Longrightarrow-\Delta \leq s(k+1)<s(k) \\
s(k)<-\Delta \Longrightarrow s(k)<s(k+1) \leq \Delta \\
|s(k)| \leq \Delta \Longrightarrow|s(k+1)| \leq|s(k)| \leq \Delta
\end{gathered}
$$

Theorem 5. Definition 4 shows that the system state will cross the sliding surface $s(k)=0$ in each successive control step. In order to easily understand the reaching condition, the conditions (8)-(10) can be replaced by the ones in [26]: (1) $s(k)>\Delta \Longrightarrow 0<s(k+1)<s(k),(2) s(k)<-\Delta \Longrightarrow s(k)<$ $s(k+1)<0$.

\section{Design of Quasi-Sliding Mode Control}

In this section, we proposed a new reaching law that satisfies the reaching law conditions (8)-(10). This new reaching law is essentially different from the conventional reaching law, due to the fact that it adopts a piece-wise reaching law.

Assuming the matched uncertainty $d(k)=0$, system (3) can be written as follows:

$$
x(k+1)=G x(k)+\Gamma u(k)
$$

For system (11) we proposed a new reaching law:

$$
s(k+1)=(1-q T) s(k)-\varepsilon T w[\Delta, s(k)]
$$

where $q$ is convergence rate parameter $(q>0), 1-q T>0$, $\varepsilon>0$ and $T$ is the sampling period. The function $w[\Delta, s(k)]$, where $\Delta, s(k) \in \mathrm{R}$, is described by

$$
w[\Delta, s(k)]= \begin{cases}\operatorname{sign}[s(k)], & |s(k)|>\Delta \\ \tan \left(\frac{\pi s(k)}{4 \Delta}\right), & |s(k)| \leq \Delta\end{cases}
$$

In order to describe it easily, $w[\Delta, s(k)]$ is written as $w$.

To obtain the control law, substituting $s(k)$ from (4) into (12) yields

$$
C^{T} x(k+1)=(1-q T) s(k)-\varepsilon T w
$$

Solving for $u(k)$ gives the control law:

$$
\begin{aligned}
& u(k) \\
& \quad=-\left(C^{T} \Gamma\right)^{-1}\left[(1-q T)\left(s(k)-\varepsilon T w-C^{T} G x(k)\right)\right]
\end{aligned}
$$

In order to ensure that the discrete-time control system designed by reaching law (12) satisfies the reaching conditions (8)-(10) and improve the performance of the reaching phase at the same time, selecting appropriate value of parameters in reaching law (12) is necessary. The parameters include $q, T, \varepsilon$, $\Delta$.

Theorem 6. Consider reaching conditions (8)-(10), another reaching condition has similar function as (8)-(10) in judging the convergence of system trajectory towards QSMD; its formulation is as follows:

$$
\begin{aligned}
& {[s(k+1)+s(k)] \operatorname{sign}[s(k)]>0} \\
& {[s(k+1)-s(k)] \operatorname{sign}[s(k)]<0}
\end{aligned}
$$

If $\varepsilon / \Delta<(2-q T) / T$, the discrete-time control system designed by the reaching law (12) satisfies the reaching conditions (16)(17).

Proof. For any $s(k) \neq 0$, we obtain

$$
\begin{aligned}
& {[s(k+1)-s(k)] \operatorname{sign}[s(k)]=-q T|s(k)|-\varepsilon T|w|} \\
& \quad<0 \\
& {[s(k+1)+s(k)] \operatorname{sign}[s(k)]} \\
& \quad=[(2-q T) s(k)-\varepsilon T w] \operatorname{sign}[s(k)]
\end{aligned}
$$

When $|s(k)|>\Delta$, (19) can be written as follows:

$$
\begin{aligned}
& {[s(k+1)+s(k)] \operatorname{sign}[s(k)]=(2-q T)|s(k)|-\varepsilon T} \\
& \quad>0
\end{aligned}
$$

When $|s(k)|<\Delta$, (19) can be rewritten as follows:

$$
\begin{aligned}
& {[s(k+1)+s(k)] \operatorname{sign}[s(k)]} \\
& \quad>\left(2-q T-\frac{\varepsilon T \pi}{4 \Delta}\right)|s(k)|>0
\end{aligned}
$$


If $(2-q T) / T>\varepsilon / \Delta$, we can obtain a conclusion that the discrete-time control system designed by the reaching law (12) satisfies the reaching conditions (16)-(17); namely, $\mid s(k+$ $1)|<| s(k) \mid$.

Theorem 7. If $(1-q T) / T<\varepsilon / \Delta<(2-q T) / T$, the discrete-time control system is controlled by a new QSM control law which is driven by reaching law (12); the system state trajectory distance from the $\Delta$-vicinity of the switching surface monotonously decreases with $k$ when $|s(k)|>\varepsilon T /(1-q T)$.

Proof. By noting Theorem 6, when $(1-q T) / T<\varepsilon / T, \varepsilon T /(1-$ $q T)>\Delta$, and then $|s(k)|>\varepsilon T /(1-q T)$; i.e., $|s(k)|>\Delta$; then $w=\operatorname{sign}[s(k)]$; the reaching law (12) can be modified as follows:

$$
\begin{aligned}
s(k+1) & =(1-q T) s(k)-\varepsilon T w \\
& =\left(1-q T-\frac{\varepsilon T}{|s(k)|}\right) s(k)
\end{aligned}
$$

Because $0<(1-q T)<1$ and $\varepsilon T /|s(k)|>0$, so $(1-q T-$ $\varepsilon T /|s(k)|)<1$, and because $|s(k)|>\varepsilon T /(1-q T)$, so $(1-q T-$ $\varepsilon T /|s(k)|)>0$, and then $0<(1-q T-\varepsilon T /|s(k)|)<1$; from the reaching conditions (8)-(10), we can obtain a conclusion that $|s(k+1)|<|s(k)|$, and the system trajectory will approach the switching surface gradually.

Theorem 8. If $\left|s\left(k_{0}\right)\right|=\varepsilon T /(1-q T)$, where $k_{0}$ is a positive constant, the control system is an ideal sliding mode control system. Since the disturbance $d(k)=0$, the system state trajectory will track along the sliding surface $s(k)=0$ towards zero point without chattering; when $0<|s(k)|<\varepsilon T /(1-q T)$, the control system is called quasi-sliding mode control system.

Proof. If $\left|s\left(k_{1}\right)\right|=\varepsilon T /(1-q T)$, where $k_{1}$ is a positive constant, the proposed reaching law can be expressed as follows:

$$
|s(k+1)|=\left(1-q T-\frac{\varepsilon T}{\left|s\left(k_{1}\right)\right|}\right)=0
$$

The system trajectory will travel along the switching surface without chattering after $k_{1}$.

If $|s(k)| \neq \varepsilon T /(1-q T)$, when $\Delta<|s(k)|<\varepsilon T /(1-q T)$, then $(1-q T-\varepsilon T /(1-q T))<0$, and because $\varepsilon / T<(2-q T) / T$, then $(1-q T-\varepsilon T /|s(k)|)>-1,-1<1-q T-\varepsilon T /|s(k)|<0$. It indicates that $\operatorname{sign}[s(k+1)]=-\operatorname{sign}[s(k)]$, and once the state trajectory first crossed the sliding surface, the system trajectory will cross the sliding surface in each subsequent step.

If $|s(i)|<\Delta, i=0,1, \cdots, k$, the following deduction is generated:

$$
\begin{aligned}
s(k+1) & =(1-q T) s(k)-\varepsilon T \tan \left[\frac{\pi s(k)}{4 \Delta}\right] \\
& \leq\left(1-q T-\frac{\varepsilon \pi T}{4 \Delta}\right) s(k) \leq \cdots \\
& \leq\left(1-q T-\frac{\varepsilon \pi T}{4 \Delta}\right)^{k+1} s(0)
\end{aligned}
$$

where $s(0)$ is the initial state of $s(k)$.
Because $\varepsilon>0, q>0$, and $\Delta>0$, so $(1-q T-\varepsilon T / \Delta)<1$ and, then $(1-q T-\varepsilon \pi T / 4 \Delta)<1$, and because $\varepsilon / \Delta<(2-$ $q T) / T$, so $\varepsilon \pi / 4 \Delta<(2-q T) / T$ and, then $(1-q T-\varepsilon T \pi / 4 \Delta)>$ $-1,-1<1-q T-\varepsilon \pi T / 4 \Delta<1$. By noting Theorem 5 , when $\varepsilon / \Delta<(2-q T) / T$, then $|s(k+1)|<|s(k)|$, if $k \longrightarrow+\infty$, $|s(k+1)| \longrightarrow 0$.

\section{Control Stability for Uncertain System}

Section 3 describes control strategy for a nominal system. However, nonnegligible parameter uncertainties and external disturbance will occur in practical systems. Therefore, we designed a new reaching law for uncertain systems based on the reaching law (12). We proposed the following reaching law for the uncertain system described by (3)

$$
\begin{aligned}
s(k+1)= & (1-q T) s(k)-\varepsilon T w+d_{s}(k)-d_{1} \\
& -d_{2} \operatorname{sign}[s(k)]
\end{aligned}
$$

Using the reaching law, solving for the control law:

$$
\begin{aligned}
& u(k)=\left(C^{T} \Gamma\right)^{-1}\left\{(1-q T) s(k)-\varepsilon T w-C^{T} A x(k)\right. \\
& \left.-d_{1}-d_{2} \operatorname{sign}[s(k)]\right\}
\end{aligned}
$$

We can know from (6), for any $k$, that

$$
\left|d_{s}(k)-d_{1}\right| \leq d_{2}
$$

This indicates that for any nonzero value of $s(k)$

$$
\operatorname{sign}\left\{\left[d_{s}(k)-d_{1}-d_{2} \operatorname{sign}[s(k)]\right\}=-\operatorname{sign}[s(k)]\right.
$$

Thus, the reaching law (25) satisfies the reaching conditions (8)-(10), and then, for any initial point, the reaching law (25) will ensure that system state will approach the switching surface $s(k)=0$ as fast as possible.

In Section 3, we have demonstrated that for the reaching law (12), when $|s(k)|<\Delta$, the sliding variable $s(k)$ will cross the switching surface in each successive step. Taking (28) into account, we conclude that it holds true for reaching law (25). The next task is to choose suitable parameter values to alleviate the influence of uncertainty for the system state. The parameters include $q, \varepsilon$, and $\Delta$.

Remark 9. When $|s(k)|<\Delta$, the reaching law (25) can be modified as follows:

$$
\begin{aligned}
s(k+1)= & (1-q T) s(k)-\varepsilon T \tan \left(\frac{\pi s(k)}{4 \Delta}\right)+d_{s}(k) \\
& -d_{1}-d_{2} \operatorname{sign}[s(k)]
\end{aligned}
$$

This has the following properties:

$$
\begin{aligned}
& s(k)>2 d_{2}+\varepsilon T \tan \left(\frac{\pi s(k)}{4 \Delta}\right) \Longrightarrow \\
& -2 d_{2}-\varepsilon T \tan \left(\frac{\pi s(k)}{4 \Delta}\right)<s(k+1)<s(k)
\end{aligned}
$$




$$
\begin{aligned}
& s(k)<-2 d_{2}-\varepsilon T \tan \left(\frac{\pi s(k)}{4 \Delta}\right) \Longrightarrow s(k)<s(k+1) \\
& <2 d_{2}+\varepsilon T \tan \left(\frac{\pi s(k)}{4 \Delta}\right) \\
& |s(k)|<2 d_{2}+\varepsilon T \tan \left(\frac{\pi s(k)}{4 \Delta}\right) \Longrightarrow|s(k+1)| \\
& <|s(k)|
\end{aligned}
$$

We can know from the reaching law (29) that although it yields a bigger quasi-sliding width than the reaching law proposed in [33], the reaching law (29) is decreased with time; when $k \longrightarrow+\infty$, then the reaching law (29) has the same quasi-sliding width $2 d_{2}$. It is easily seen that the controller (26) can ensure that the proposed reaching law (25) satisfies the reaching condition (30). Thus, a QSM within the $2 d_{s}(k)$ neighbourhood of surface $s(k)=0$ can be attained. However, the reaching law proposed in $[33,42]$ has two shortcomings: one is that it has larger amplitude of chattering caused by disturbance than the proposed reaching law (25); another one is that the reaching laws proposed in $[33,42]$ require that the rate of change of uncertainty is tremendously slow, but the condition rarely occurs in practical systems. The result will be validated by a numerical simulation in Section 5 .

Theorem 10. Assuming the uncertainty $d_{s}(k)$ satisfies the equation $d_{s}(k)=-d_{s}(k+1)+o\left(T^{2}\right)$, the reaching law (25) will be influenced slightly by disturbance $d_{s}(k)$ when system trajectory enters the $\Delta$-neighbourhood of the designed sliding surface $s(k)=0$, and it will converge to zero in finite time.

Proof. (1) If $0<s(j)<\Delta, j=0,1,2, \cdots, k$.

$$
\begin{aligned}
& \mathrm{s}(1)=(1-\mathrm{qT}) \mathrm{s}(0)-\varepsilon T \psi(0)+\varphi(0) \\
& \mathrm{s}(2)=(1-\mathrm{qT}) \mathrm{s}(1)-\varepsilon T \psi(1)+\varphi(1)
\end{aligned}
$$

$$
\begin{aligned}
& =(1-q T)^{2} s(0)-(1-q T)(\varepsilon T \psi(0)-\varphi(0)) \\
& -(\varepsilon T \psi(1)-\varphi(1)) \\
s(k) & =(1-q T)^{k} s(0) \\
& -\sum_{j=0}^{k-2}(1-q T)^{k-j-1}(\varepsilon T \psi(j)-\varphi(j)) \\
& -[\varepsilon T \psi(k-1)-\varphi(k-1) \\
= & (1-q T)^{k} s(0) \\
& -\sum_{j=0}^{k-2}(1-q T)^{k-j-1}\left[\varepsilon T \tan \left(\frac{\pi s(j)}{4 \Delta}\right)-\varphi(j)\right] \\
& -\left[\varepsilon T \tan \left(\frac{\pi s(k-1)}{4 \Delta}\right)-\varphi(k-1)\right] \\
\leq & (1-q T)^{k} s(0) \\
& -\sum_{j=0}^{k-1}(1-q T)^{k-j-1}\left[\varepsilon T \tan \left(\frac{\pi s(k)}{4 \Delta}\right)\right]
\end{aligned}
$$

Assume there exists a real number $n$ that satisfies the following equation:

$$
\begin{gathered}
\sum_{j=0}^{k-1}(1-q T)^{k-j-1}\left[\varepsilon T \tan \left(\frac{\pi s(k)}{4 \Delta}\right)\right] \\
=\sum_{j=0}^{k-1}(1-q T)^{k-j-1}(\varepsilon T n)
\end{gathered}
$$

Generating the expression of $n$ gives

$$
n=\frac{(1-q T)^{k-1}(\tan (\pi s(0) / 4 \Delta))+(1-q T)^{k-2}(\tan (\pi s(1) / 4 \Delta))+\cdots+\tan (\pi s(k-1) / 4 \Delta)}{(1-q T)^{k-1}+(1-q T)^{k-2}+\cdots+1}
$$

where $s(0)$ is any initial point of $s(k), \psi(j)=\tan (\pi s(j) / 4 \Delta)$, $\varphi(j)=d_{s}(j)-d_{1}-d_{2} \operatorname{sign}[s(j)]$.

Since $0<s(j)<\Delta$, then $0<\tan (\pi s(j) / 4 \Delta)<1$, according to (33), the following deduction can be obtained as follows:

$$
0<n<1
$$

Substituting (32) into (31), we can get a conclusion as follows:

$$
s(k) \leq(1-q T)^{k} s(0)-\sum_{j=0}^{k-1}(1-q T)^{k-j-1}(\varepsilon T n)
$$

$$
\begin{aligned}
& =(1-q T)^{k} s(0)-\frac{1-(1-q T)^{k}}{q T} \varepsilon T n \\
& =\frac{(1-q T)^{k} s(0) q T-\left(1-(1-q T)^{k} \varepsilon T n\right.}{q T}
\end{aligned}
$$

We can find a real number $k^{*}$ as follows:

$$
k^{*}=\log _{(1-q T)} \frac{\varepsilon T n^{*}}{\varepsilon T n^{*}+s(0) q T}
$$


Substituting (36) into (35), the following conclusion can be obtained:

$$
\begin{aligned}
& s\left(k^{*}\right) \\
& \leq \frac{\left(\varepsilon T n^{*} /\left(\varepsilon T n^{*}+q T s(0)\right)\right)\left[s(0) q T+\varepsilon T n^{*}\right]-\varepsilon T n^{*}}{q T} \\
& =0
\end{aligned}
$$

where $n^{*}=\left((1-q T)^{k^{*}-1}(\tan (\pi s(0) / 4 \Delta))+(1-\right.$ $\left.q T)^{k^{*}-2}(\tan (\pi s(1) / 4 \Delta))+\cdots+\tan \left(\pi s\left(k^{*}-1\right) / 4 \Delta\right)\right) /$ $\left((1-q T)^{k^{*}-1}+(1-q T)^{k^{*}-2}+\cdots+1\right)$

(2) If $-\Delta<s(i)<0, i=0,1,2, \cdots k$, considering Theorem 7 and (25) and (31), the following deduction can be obtained:

$$
\begin{aligned}
s(k) & \\
= & (1-q T)^{k} s(0) \\
& -\sum_{j=0}^{k-2}(1-q T)^{k-j-1}(\varepsilon T \psi(j)-\varphi(j)) \\
& -[\varepsilon T \psi(k-1)-\varphi(k-1)] \\
= & 1-q T)^{k} s(0) \\
& -\sum_{j=0}^{k-2}(1-q T)^{k-j-1}\left(\varepsilon T \tan \left(\frac{\pi s(j)}{4 \Delta}\right)-\varphi(j)\right) \\
& -\left[\varepsilon T \tan \frac{\pi s(k-1)}{4 \Delta}-\varphi(j-1)\right] \\
\geq & (1-q T)^{k} s(0) \\
& -\sum_{j=0}^{k-1}(1-q T)^{k-j-1}\left(\varepsilon T \tan \left(\frac{\pi s(j)}{4 \Delta}\right)\right)
\end{aligned}
$$

Similar to the derivation from (32) to (35), there exists a real number $k^{*}$ as follows:

$$
k^{*}=\log _{(1-q T)} \frac{\varepsilon T n^{*}}{\varepsilon T n^{*}+s(0) q T}
$$

Substituting (39) into (38), the conclusion can be obtained:

$$
\mathrm{s}\left(k^{*}\right) \geq 0
$$

Through the above analysis, we can obtain the conclusion that the $s(k)$ will first cross the sliding surface within $k=\left\lfloor k^{*}\right\rfloor+1$ steps at most, where the $\left\lfloor k^{*}\right\rfloor$ denotes the maximal integer bounded below the real number $k^{*}$. By noting reaching conditions (8)-(10), once the variable $s(k)$ reaches the sliding surface, it crosses it again in each successive step.

According to (37) and (40), when a discrete-time system has a step uncertainty, i.e., $d_{s}(k)=-d_{s}(k+1)+\mathrm{o}\left(T^{2}\right)$, the proposed reaching law possesses excellent properties; it will force the system states to enter the quasi-sliding mode manifold; once the representative points enter the QSM manifold, it never escapes from it.
Theorem 11. From Theorem 10, we know that every two adjacent disturbance signs are different, if the disturbance signal is input into system (3), the value of variable $|s(k)|$ in [42] cannot converge to zero, but only to remain always a periodic fluctuation around sliding surface $s(k)=0$.

Proof. When $|s(k)|<\Delta$,

$$
\begin{aligned}
& |s(1)|=(1-q T) s(0)-\frac{\lambda}{\Psi(0)}+F(0) \\
& |s(2)|=(1-q T) s(1)-\frac{\lambda}{\Psi(1)}+F(1)=(1-\mathrm{qT}) \\
& . \Psi(1)\left[(1-\mathrm{qT}) \Psi(0) \mathrm{s}(0)-\frac{\lambda}{\Psi(0)}+\mathrm{F}(0)\right] \\
& -\frac{\lambda}{\Psi(1)}+\mathrm{F}(1)=(1-q T)^{2} \Psi(0) \Psi(1) s(0)-(1 \\
& -q T) \Psi(1) \times\left[\frac{\lambda}{\Psi(0)}-F(0)\right]-\left[\frac{\lambda}{\Psi(1)}-F(1)\right] \\
& |s(k)|=(1-q T)^{k} s(0) \prod_{i=0}^{k} \Psi(i) \\
& -\sum_{j=0}^{k-2}\left[(1-q T)^{k-i-1}[\Psi(k-1) \times \cdots \times \Psi(i+1)]\right] \\
& \times\left[\frac{\lambda}{\Psi(i)}-F(i)\right]-\left[\frac{\lambda}{\Psi(k-1)}-F(k-1)\right] \\
& \leq(1-q T)^{k} s(0) \Psi_{1} \\
& -\Psi_{2}\left\{\sum_{i=0}^{k-2}(1-q T)^{k-i-1}\left[\frac{\lambda}{\Psi(i)}-F(i)\right]\right. \\
& \left.+\left[\frac{\lambda}{\Psi(k-1)}-F(k-1)\right]\right\} \\
& =(1-q T)^{k} s(0) \Psi_{1} \\
& -\Psi_{2}\left\{\sum_{i=0}^{k-1}(1-q T)^{k-1-i}\left[\frac{\lambda}{\Psi(i)}-F(i)\right]\right\}
\end{aligned}
$$

For clarification, relevant equations can be written as follows: $\Psi_{1}=\prod_{i=0}^{k-1} \Psi(i), \Psi_{2}=[\Psi(k-1) \times \cdots \times \Psi(i+1)], F=$ $C\left[d_{s}(k)-2 d_{s}(k-1)+d_{s}(k-2)\right], \Psi(i)=\sigma+(1-\sigma) e^{-\varphi|s(k)|^{\gamma}}$, where $0<\sigma<1, \gamma \in \mathrm{N}$.

Similar to the derivation from (32) to (35), there exists a real number $m$ that makes $\sum_{i=0}^{k-1}(1-q T)^{k-1-i}[\lambda / \Psi(i)]=$ $\sum_{i=0}^{k-1}(1-q T)^{k-1-i}[\lambda / m]$; the following conclusion can be derived:

$$
\begin{aligned}
s(k) \leq & (1-q T)^{k} s(0) \Psi_{1} \\
& -\Psi_{2}\left\{\sum_{i=0}^{k-1}(1-q T)^{k-1-i}\left[\frac{\lambda}{m}-\phi\right\}\right.
\end{aligned}
$$


By noting Theorem $10,\left|4 d_{s}(k)+O\left(T^{2}\right)\right|=\mid d_{s}(k)-2 d_{s}(k-$ $1)+d_{s}(k-2) \mid$, where $\left|4 d_{s}(k)+O\left(T^{2}\right)\right|=\phi$.

Then, we can find a real number $k^{*}$ :

$$
k^{*}=\log _{(1-q T)} \frac{\lambda / m^{*}-\phi^{*}}{s(0)\left(\Psi_{1}^{*} / \Psi_{2}^{*}\right) q T+\lambda / m^{*}-\phi^{*}}
$$

where $\Psi_{1}^{*}=\prod_{i=0}^{k^{*}-1} \Psi(i), \Psi_{2}^{*}=\prod_{i=0}^{k^{*}-1} \Psi(i), m^{*}=((1-$ $\left.q T)^{k^{*}-1}+(1-q T)^{k^{*}-1+\cdots+1}\right) /\left((1-q T)^{k^{*}-1} / \Psi(0)+(1-q T)^{k^{*}-2} /\right.$ $\left.\Psi(1)+\cdots+1 / \Psi\left(k^{*}-1\right)\right)$, and $\phi^{*}=\left|4 d_{s}\left(k^{*}\right)+O\left(T^{2}\right)\right|$.

Substituting (43) into (42), the following conclusion can be obtained:

$$
\left|s\left(k^{*}\right)\right| \longrightarrow 0
$$

The aforementioned case shows that within $k_{1}=\left\lfloor k^{*}\right\rfloor+1$ steps at most, the variable $s(k)$ from any initial point will first cross the sliding surface. Although the variable $s(k)$ can cross sliding surface $s(k)=0$ in $k^{*}$, the value of $|s(k)|$ cannot stay a stable value in subsequent steps. After $k^{*}$ the value of $s(k)$ will increases with $k$, then it will approach a maximum boundary, which is determined by $d_{s}(k)$. Similar to the derivation (41) to (44), there exists a real number $k_{2}^{*}$ which makes variable $s\left(k_{2}^{*}\right) \longrightarrow 0$, where $k_{2}^{*}>k^{*}$. A conclusion can be obtained that the variable $s(k)$ trajectory in [42] is a periodic fluctuation within sliding mode manifold; the results will be verified in Section 5 .

\section{Numerical Simulation}

In this section, we will demonstrate the effectiveness of the proposed method by means of a simulation example. We will compare the control law proposed by this paper with the method proposed in [42].

Consider the discrete-time system (3) with parameters as follows:

$$
\begin{aligned}
G & =\left[\begin{array}{cc}
1 & 0.01 \\
0 & 0.7
\end{array}\right], \\
\Gamma & =\left[\begin{array}{c}
0.01 \\
1
\end{array}\right], \\
d_{s}(k) & =\left[\begin{array}{c}
0 \\
0.1 *(-1)^{\wedge}\left(\frac{k}{9}\right)
\end{array}\right]
\end{aligned}
$$

The control law proposed by [42] is as follows:

$$
\begin{aligned}
& u(k)=-\left(C^{T} \Gamma\right)^{-1}\left[C^{T} G x(k)-(1-q T) \Psi(k) s(k)\right. \\
& +2 C^{T} d_{s}(k-1)-C^{T} d_{s}(k-2) \\
& \left.+\frac{\lambda}{\Psi(\mathrm{k})} \operatorname{sign}(s(k))\right]
\end{aligned}
$$
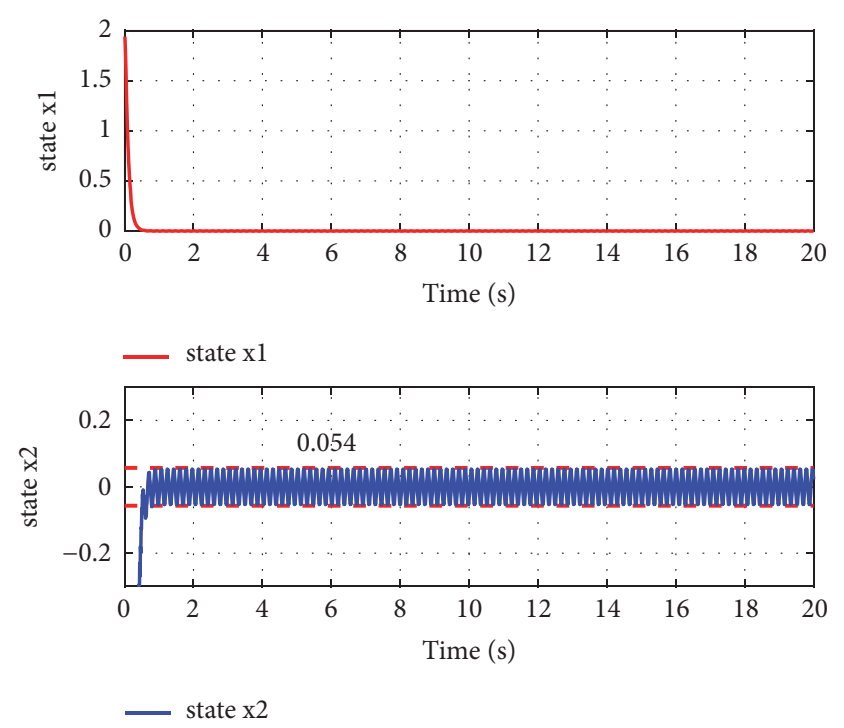

FIGURE 1: Evolution of system states with the proposed reaching law.

where

$$
\begin{aligned}
\Psi(k)= & \\
& +(1-\sigma) e^{-\varphi|s(k)|^{\gamma}} \begin{cases}0<\sigma<1, & \varphi>0 \\
\gamma>0, & \text { and } \gamma \in N\end{cases}
\end{aligned}
$$

Vector $C$ needs to satisfy the conditions that $C^{T} \Gamma \neq 0$ and the eigenvalues of the matrix $\left(I-\Gamma\left(C^{T} \Gamma\right)^{-1} C^{T}\right) G$ lie in the left of imaginary plane, so the sliding vector can be set $C^{T}=$ [10 1]; for the reaching law proposed in [42], $q=50, \lambda=$ $0.16, \sigma=0.65, \gamma=10, \varphi=20$, and $T=0.01 s$; the initial state is set as $x(0)=[2,-6]$. The values of $q, \varepsilon, T$, and $\Delta$ satisfy the condition $(1-q T) / T<\varepsilon / \Delta<(2-q T) / T$, for the reaching law (25), $q=10, \varepsilon=240, \Delta=1.1$, and $T=0.01 s$.

Figures 1 and 2 exhibit the evolution of system state trajectories which are controlled by control law (26) and (46), respectively. In the simulation example, the system state $x_{1}$ is not be affected by disturbance $d_{s}(\mathrm{k})$, so the control laws (26) and (46) are derived from reaching law (12) and [42] can force system state $x_{1}$ to converge to zero from any initial point. It can be observed that the discrete-time system has disturbance $d_{s}(k)$ where the rate of change is fast, by noting (37) and (40) and reaching conditions (8)-(10); if system state $x_{1}$ enters the $\Delta$-vicinity of switching surface and then converges to zero in finite time, the switching function $s(k)$ will force the system state $x_{2}$ to converge to sliding surface in finite time; the amplitude of chattering is 0.054 , as shown in Figure 1. However, by noting (44) and reaching conditions (8)-(10), $s(k)$ cannot converge to zero but form a periodic fluctuation; then $s(k)$ will force the evolution curve of system state $x_{2}$ to exhibit periodic fluctuation; the amplitude of chattering is much larger than the state $x_{2}$ which is controlled by control law (26), as shown in Figure 2.

The discrete-time system (3) has disturbance $d_{s}(k)$ in which the rate of change is high. The minimum width of QSMD in [42] can be obtained, $\Delta_{43}=0.14$, as shown in 

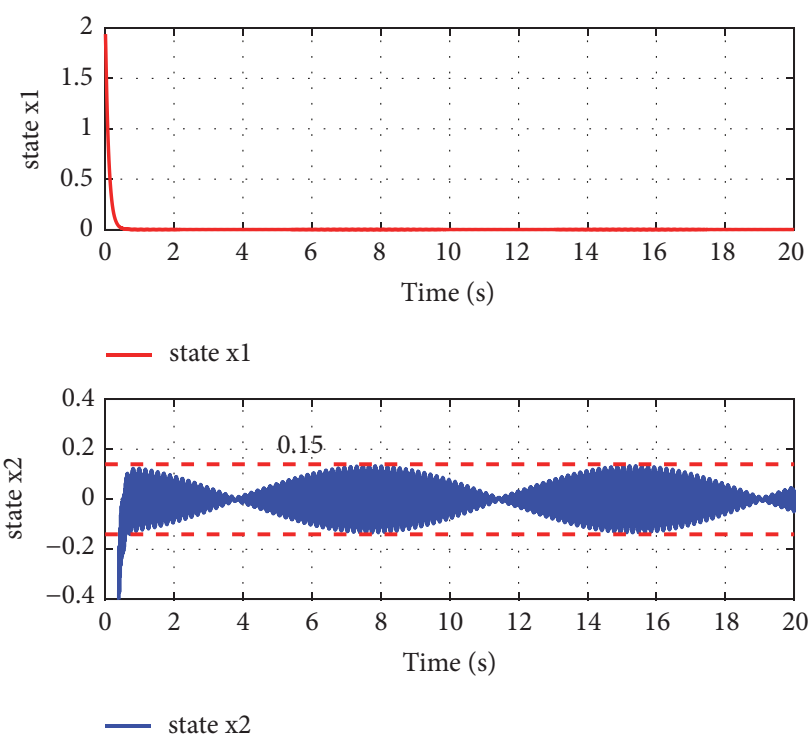

FIGURE 2: Evolution of system states with the reaching law [42].

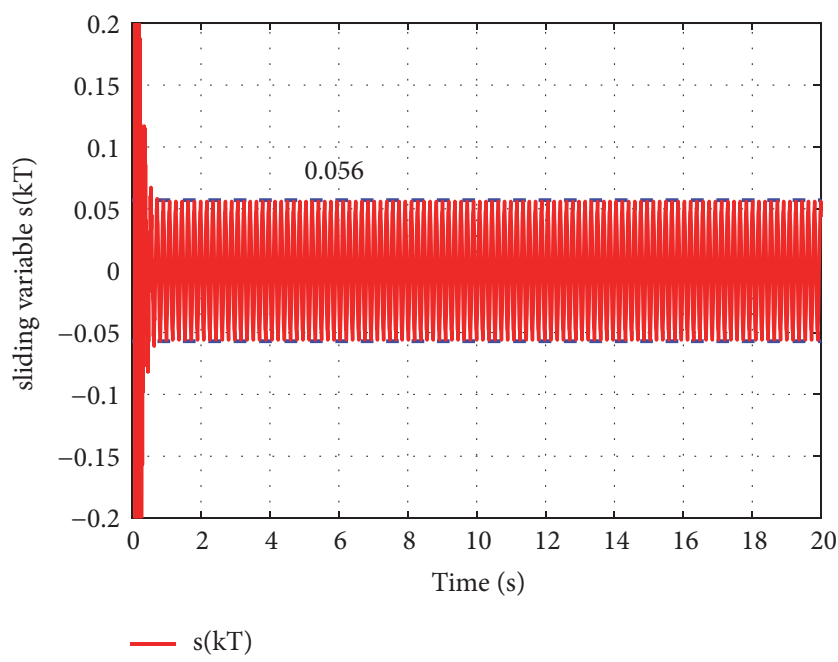

FIGURE 3: Evolution of sliding variable $s(k)$ with the proposed reaching law.

Figure 4. The minimum width of QSMD in the proposed method is $\Delta=0.056$, as shown in Figure 3, which is much smaller than $\Delta_{43}$. From Figure 4 , one can easily see that the simulation result confirms Theorem 11.

The control laws (26) and (46) are derived from the proposed reaching law in this paper and the reaching law in [42], respectively. By noting Theorem 11 and (44), the variable $s(k)$ cannot converge to a stable value but exhibits a periodic fluctuation; then the evolution curve of system control signal also is a period fluctuation, as shown in Figure 6; the amplitude of control signal is $a_{1}=0.31$. However, by noting Theorem 10, (37), and (40), the variable $s(k)$ can converge to zero, and the amplitude of control signal is $a_{2}=0.095$, as shown in Figure 5, which is much smaller than $a_{1}$.

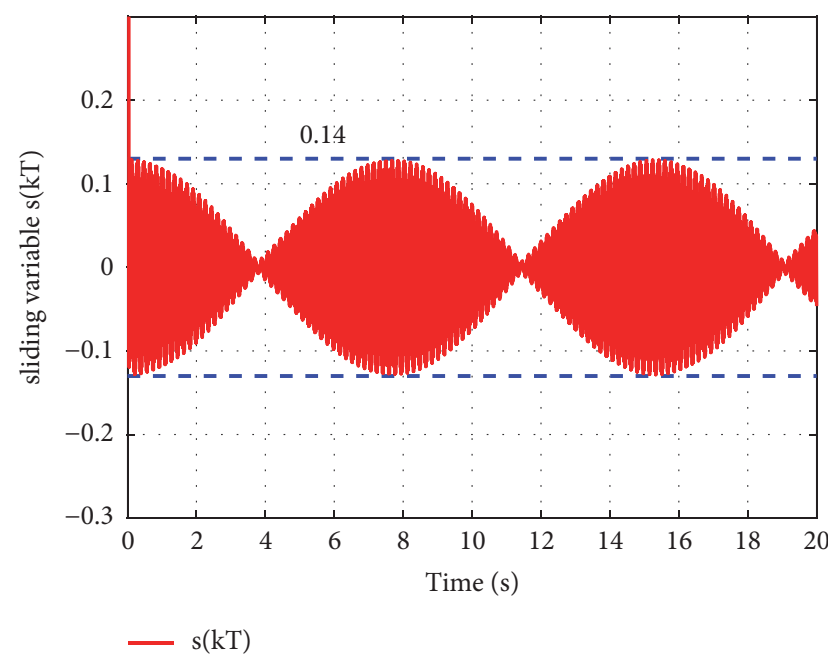

FIGURE 4: Evolution of sliding variable $s(k)$ with the reaching law [42].

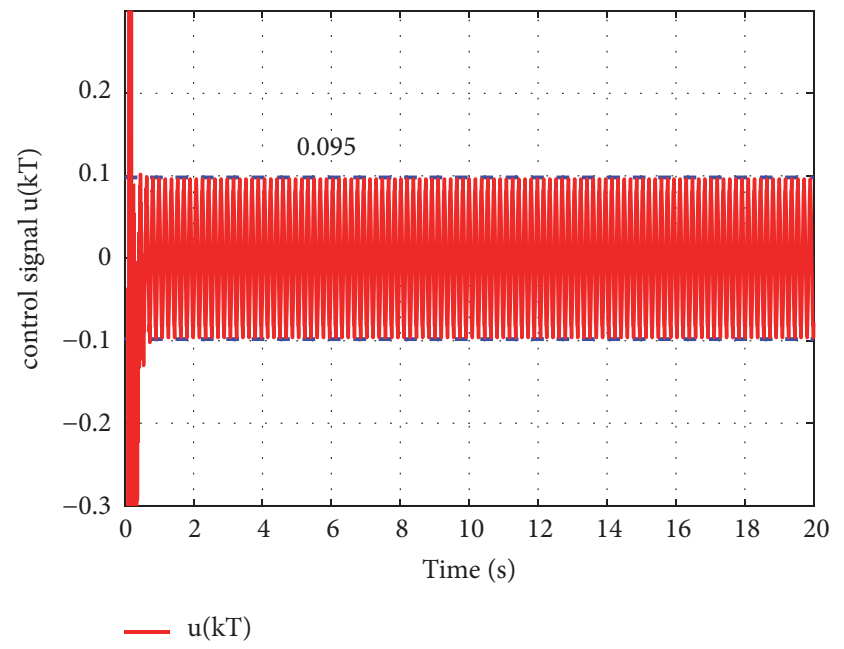

FIGURE 5: Evolution of control signal with the proposed reaching law.

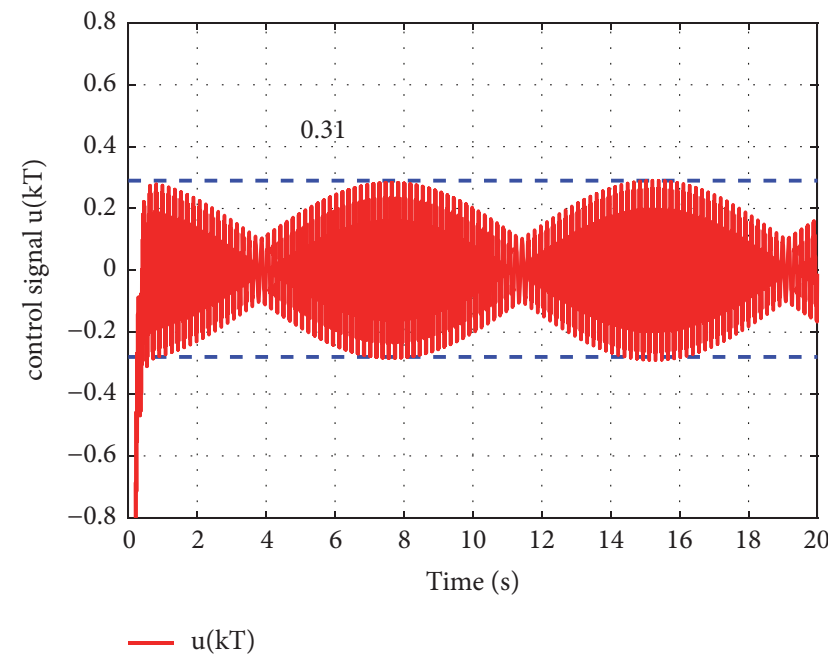

FiguRE 6: Evolution of control signal with the reaching law [42]. 


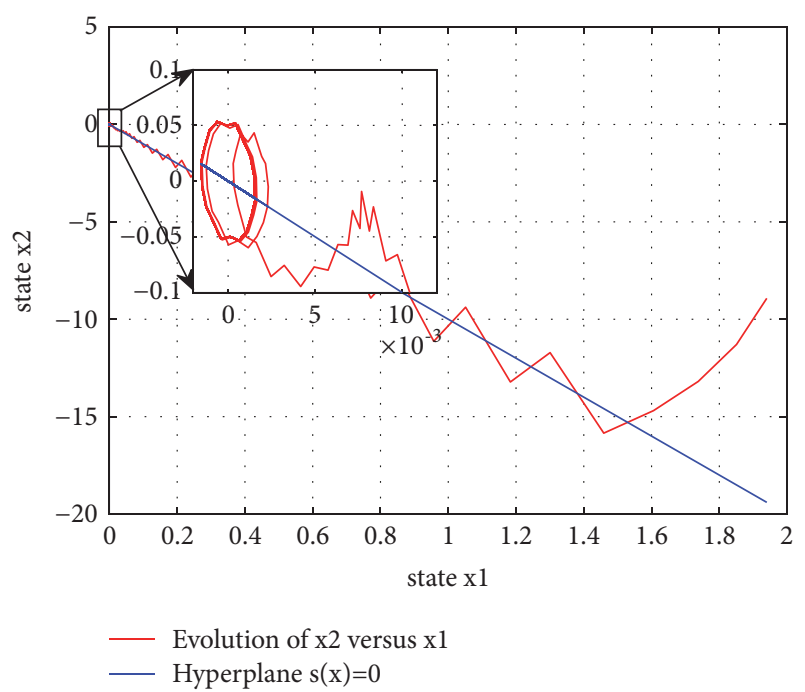

Figure 7: Phase plane diagram of $x_{2}(k T)$ versus $x_{1}(k T)$ with the proposed reaching law.

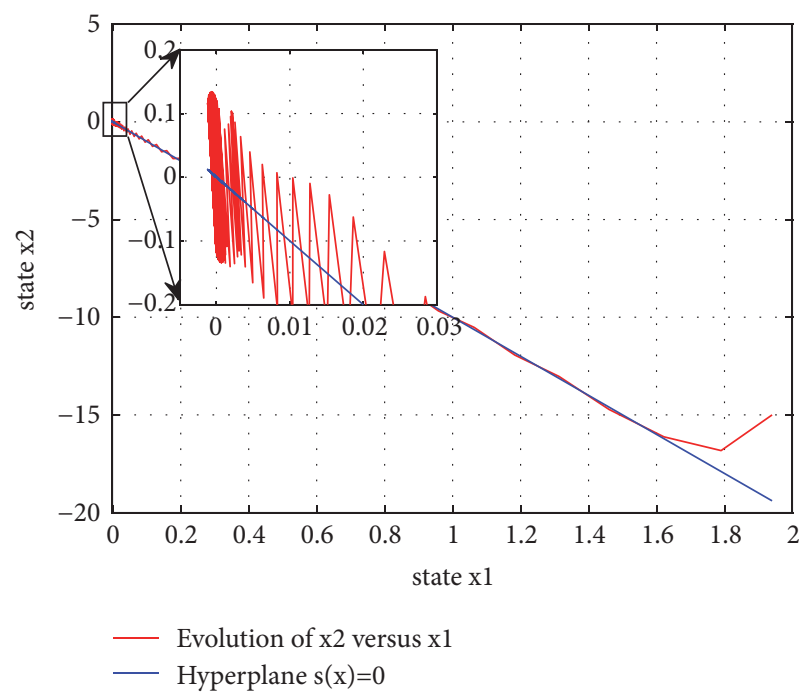

FIgURE 8: Phase plane diagram of $x_{2}(k T)$ versus $x_{1}(k T)$ with the reaching law [42].

Figures 7 and 8 exhibit the phase plane diagram of $x_{2}$ versus $x_{1}$ with proposed reaching law and reaching law in [42], respectively. It can be observed that both control laws (26) and (46) are able to force system states $x_{1}$ and $x_{2}$ to reaching sliding surface $s(k)=0$. In Figure 7, one easily sees that the representative point $x_{1}$ first reaches sliding surface $s(k)=0$ at $x_{1} \cong 1.5$ and converges to zero in finite time; the amplitude of chattering around the zero point is about 0.05 . However, in Figure 8, the amplitude of chattering around the zero point is more than 0.1 , which is larger than the proposed reaching law. It is obvious that the control performance is effectively improved by employing the proposed reaching law.

\section{Conclusion}

In this paper, a new quasi-sliding mode reaching law is proposed, which employs a tangent function term to replace the sign function term. The comparison between the proposed strategy and an existing work [42] has been made finding that the proposed reaching law possesses excellent properties. Not only is this proposed reaching law able to alleviate chattering in the presence of disturbance $d_{s}(k)$ where the rate of change is fast, but also it guarantees the robustness of control system; the reason is that the proposed reaching law in this paper ensures that the variable $s(k)$ crosses sliding surface $s(k)=0$ in every successive step. On the other hand, the proposed method makes the amplitude of chattering of system trajectories around sliding surface $s(k)=0$ smaller than reaching law [42]. Since the proposed reaching law may apply in hard disk drive control systems, the further works mainly focus on verifying this idea.

\section{Data Availability}

The data used to support the findings of this study are available from the corresponding author upon request.

\section{Conflicts of Interest}

The authors declare that they have no conflicts of interest.

\section{Acknowledgments}

This work is partially supported by the Natural Science Foundation of China and Key Research and Development Program of China (2016YFC0301400, 51379198, 51075377, and 31202036).

\section{References}

[1] V. I. Utkin, "Sliding mode control in discrete-time and difference systems," in Variable Structure and Lyapunov Control, pp. 87-107, Springer, 1994.

[2] K. Abidi, J.-X. Xu, and X. Yu, "On the discrete-time integral sliding-mode control," IEEE Transactions on Automatic Control, vol. 52, no. 4, pp. 709-715, 2007.

[3] S. Chakrabarty and B. Bandyopadhyay, "A generalized reaching law for discrete time sliding mode control," Automatica, vol. 52, pp. 83-86, 2015.

[4] B. Bandyopadhyay and D. Fulwani, "High-performance tracking controller for discrete plant using nonlinear sliding surface," IEEE Transactions on Industrial Electronics, vol. 56, no. 9, pp. 3628-3637, 2009.

[5] S. Ding and S. Li, "Second-order sliding mode controller design subject to mismatched term," Automatica, vol. 77, pp. 388-392, 2017.

[6] S. Ding and W. X. Zheng, "Nonsingular terminal sliding mode control of nonlinear second-order systems with input saturation," International Journal of Robust and Nonlinear Control, vol. 26, no. 9, pp. 1857-1872, 2016.

[7] J. Fei, W. Yan, and Y. Yang, "Adaptive nonsingular terminal sliding mode control of MEMS gyroscope based on backstepping design," International Journal of Adaptive Control and Signal Processing, vol. 29, no. 9, pp. 1099-1115, 2015. 
[8] S. Gao, H. Dong, Y. Chen, B. Ning, G. Chen, and X. Yang, "Approximation-based robust adaptive automatic train control: an approach for actuator saturation," IEEE Transactions on Intelligent Transportation Systems, vol. 14, no. 4, pp. 1733-1742, 2013.

[9] R. Ž. Jovanović and Z. M. Bučevac, "Discrete-Time Exponentially Stabilizing Fuzzy Sliding Mode Control via Lyapunov's Method," Advances in Fuzzy Systems-Applications and Theory, vol. 2015, Article ID 496085, 10 pages, 2015.

[10] A. Bartoszewicz, "Chattering attenuation in sliding mode control systems," Control and Cybernetics, vol. 29, no. 2, pp. 585594, 2000.

[11] S. C. Garcia, M. Teixeira, J. P. F. Garcia, U. N. Alves, and J. Ribeiro, "Discrete-Time Sliding Mode Control for Uncertain Networked System Subject to Time Delay," Mathematical Problems in Engineering, vol. 2015, Article ID 195120, 10 pages, 2015.

[12] D. Savitski, D. Schleinin, V. Ivanov, and K. Augsburg, "Robust Continuous Wheel Slip Control with Reference Adaptation: Application to Brake System with Decoupled Architecture," IEEE Transactions on Industrial Informatics, 2018.

[13] B. Sun, W. Gan, M. Mei, D. Zhu, and S. X. Yang, "Cascaded UUV trajectory tracking control based on model predictive and sliding mode control," Journal of Marine Science and Technology (Taiwan), vol. 25, no. 6, pp. 671-679, 2017.

[14] E. R. Lisy, "Design of Robust Chattering-free Integral Sliding Mode Controller for DualInput Buck Boost Converter," International Journal of Applied Engineering Research, vol. 13, no. 1, pp. 358-365, 2018.

[15] T. Hu, W. Xue, and Y. Huang, "Active disturbance rejection control for permanent magnet linear motor," in Proceedings of the in Proceedings of the 31st Chinese Control Conference, pp. 296-301, Hefei, China, 2012.

[16] H.-S. Ahn, Y. Chen, and H. Dou, "State-periodic adaptive compensation of cogging and coulomb friction in permanent magnet linear motors," in Proceedings of the 2005 American Control Conference, ACC, pp. 3036-3041, USA, June 2005.

[17] A. Bartoszewicz and P. Latosiński, "Quasi-Sliding Networked Control of Systems Subject to Unbounded Disturbance with Limited Rate of Change," Mathematical Problems in Engineering, vol. 2015, Article ID 379647, 10 pages, 2015.

[18] J. Bagheri, A. Saberkari, O. Khorgami, and E. Alarcon-Cot, "Design and Implementation of a Sliding-Mode Controller for Digital Low-Dropout/Linear Regulators," IEEE Transactions on Circuits and Systems II: Express Briefs, 2018.

[19] X. Yu, B. Wang, and X. Li, "Computer-controlled variable structure systems: the state-of-the-art," IEEE Transactions on Industrial Informatics, vol. 8, no. 2, pp. 197-205, 2012.

[20] C. J. Fallaha, M. Saad, H. Y. Kanaan, and K. Al-Haddad, "Sliding-mode robot control with exponential reaching law," IEEE Transactions on Industrial Electronics, vol. 58, no. 2, pp. 600-610, 2011.

[21] Q. Xu, "Digital Integral Terminal Sliding Mode Predictive Control of Piezoelectric-Driven Motion System," IEEE Transactions on Industrial Electronics, vol. 63, no. 6, pp. 3976-3984, 2016.

[22] W. Gao and J. C. Hung, "Variable structure control of nonlinear systems. A new approach," IEEE Transactions on Industrial Electronics, vol. 40, no. 1, pp. 45-55, 1993.

[23] W. Gao, Y. Wang, and A. Homaifa, "Discrete-time variable structure control systems," IEEE Transactions on Industrial Electronics, vol. 42, no. 2, pp. 117-122, 1995.
[24] A. Bartoszewicz, "Remarks on "discrete-time variable structure control systems"," IEEE Transactions on Industrial Electronics, vol. 43, no. 1, pp. 235-238, 1996.

[25] M. C. Saaj, B. Bandyopadhyay, and H. Unbehauen, "A new algorithm for discrete-time sliding-mode control using fast output sampling feedback," IEEE Transactions on Industrial Electronics, vol. 49, no. 3, pp. 518-523, 2002.

[26] A. Bartoszewicz, "Discrete-time quasi-sliding-mode control strategies," IEEE Transactions on Industrial Electronics, vol. 45, no. 4, pp. 633-637, 1998.

[27] B. Veselic, B. Perunicic-Drazenovic, and C. Milosavljevic, "Improved discrete-time sliding mode position control using Euler velocity estimation," IEEE Transactions on Industrial Electronics, vol. 57, no. 11, pp. 3840-3847, 2010.

[28] P. Lesniewski and A. Bartoszewicz, "Inverse tangent based switching type reaching law for discrete time sliding mode control systems," in Proceedings of the European Control Conference, ECC 2015, pp. 2390-2395, Austria, July 2015.

[29] G. Golo and C. Milosavljević, "Robust discrete-time chattering free sliding mode control," Systems \& Control Letters, vol. 41, no. 1, pp. 19-28, 2000.

[30] L. Song, S. Gong, and Y. Tian, "Study of reaching law approach for discrete-time variable structure control system," in Proceedings of the 2012 International Conference on Modelling, Identification and Control, ICMIC 2012, pp. 206-210, China, June 2012.

[31] A. Bartoszewicz and P. Lesniewski, "New Switching and Nonswitching Type Reaching Laws for SMC of Discrete Time Systems," IEEE Transactions on Control Systems Technology, vol. 24, no. 2, pp. 670-677, 2016.

[32] S. C. Qu, X. H. Xia, and J. F. Zhang, "Dynamics of discrete-time sliding-mode-control uncertain systems with a disturbance compensator," IEEE Transactions on Industrial Electronics, vol. 61, no. 7, pp. 3502-3510, 2014.

[33] Y. Niu, D. W. C. Ho, and Z. Wang, "Improved sliding mode control for discrete-time systems via reaching law," IET Control Theory \& Applications, vol. 4, no. 11, pp. 2245-2251, 2010.

[34] H. Du, X. Yu, M. Z. Chen, and S. Li, "Chattering-free discretetime sliding mode control," Automatica, vol. 68, pp. 87-91, 2016.

[35] Y. S. Eun, J. H. Kim, K. S. Kim, and D. Cho, "Discretetime variable structure controller with a decoupled disturbance compensator and its application to a CNC servomechanism," IEEE Transactions on Control Systems Technology, vol. 7, no. 4, pp. 414-423, 1999.

[36] T. H. Yan, B. Wu, B. He, W. H. Li, and R. B. Wang, "A Novel Fuzzy Sliding-Mode Control for Discrete-Time Uncertain System," Mathematical Problems in Engineering, vol. 2016, Article ID 1530760, 9 pages, 2016.

[37] H. Ma, J. Wu, and Z. Xiong, "Discrete-Time Sliding-Mode Control with Improved Quasi-Sliding-Mode Domain," IEEE Transactions on Industrial Electronics, vol. 63, no. 10, pp. 6292$6304,2016$.

[38] G. Cheng and K. Peng, "Robust composite nonlinear feedback control with application to a servo positioning system," IEEE Transactions on Industrial Electronics, vol. 54, no. 2, pp. 11321140, 2007.

[39] V. Acary and B. Brogliato, "Implicit Euler numerical scheme and chattering-free implementation of sliding mode systems," Systems \& Control Letters, vol. 59, no. 5, pp. 284-293, 2010. 
[40] V. Acary, B. Brogliato, and Y. V. Orlov, "Chattering-free digital sliding-mode control with state observer and disturbance rejection," Institute of Electrical and Electronics Engineers Transactions on Automatic Control, vol. 57, no. 5, pp. 1087-1101, 2012.

[41] H. B. Oza, V. Acary, Y. V. Orlov, S. K. Spurgeon, and B. Brogliato, "Finite time tracking of unilaterally constrained planar systems with pre-specified settling time: Second order sliding mode synthesis and chattering-free digital implementation," in Proceedings of the 51st IEEE Conference on Decision and Control, CDC 2012, pp. 5471-5476, USA, December 2012.

[42] H. Ma, J. Wu, and Z. Xiong, "A novel exponential reaching law of discrete-time sliding-mode control," IEEE Transactions on Industrial Electronics, vol. 64, no. 5, pp. 3840-3850, 2017.

[43] B. Wang, X. H. Yu, and G. R. Chen, "ZOH discretization effect on single-input sliding mode control systems with matched uncertainties," Automatica, vol. 45, no. 1, pp. 118-125, 2009. 


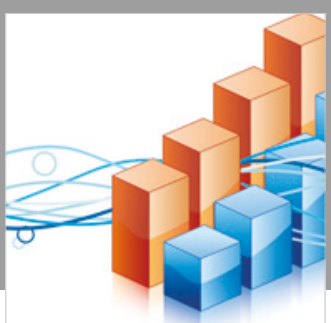

Advances in

Operations Research

\section{-n-m}
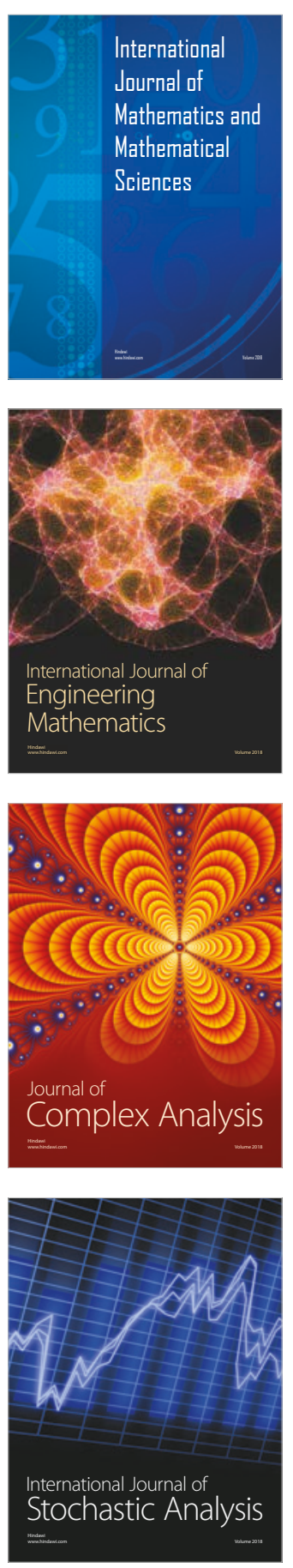
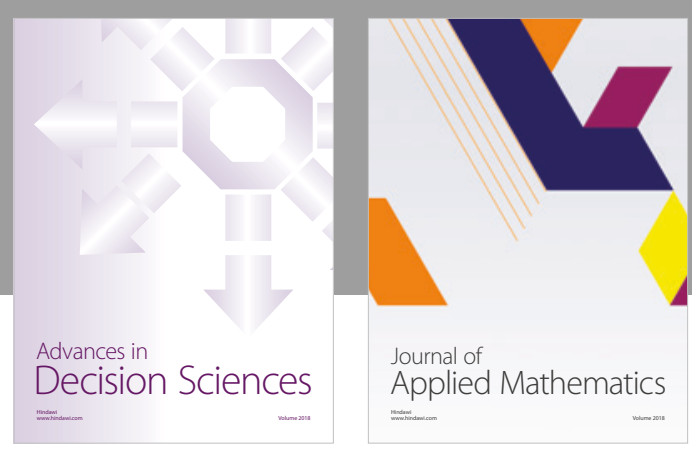

Journal of

Applied Mathematics
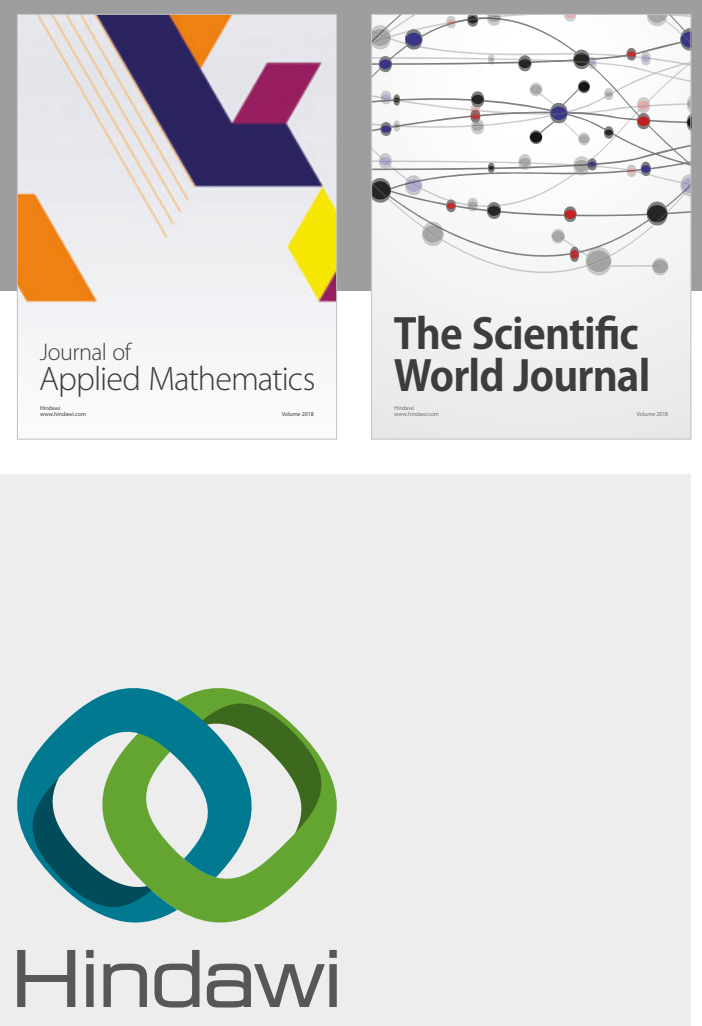

Submit your manuscripts at

www.hindawi.com

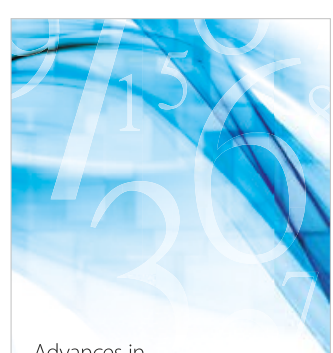

Advances in
Numerical Analysis
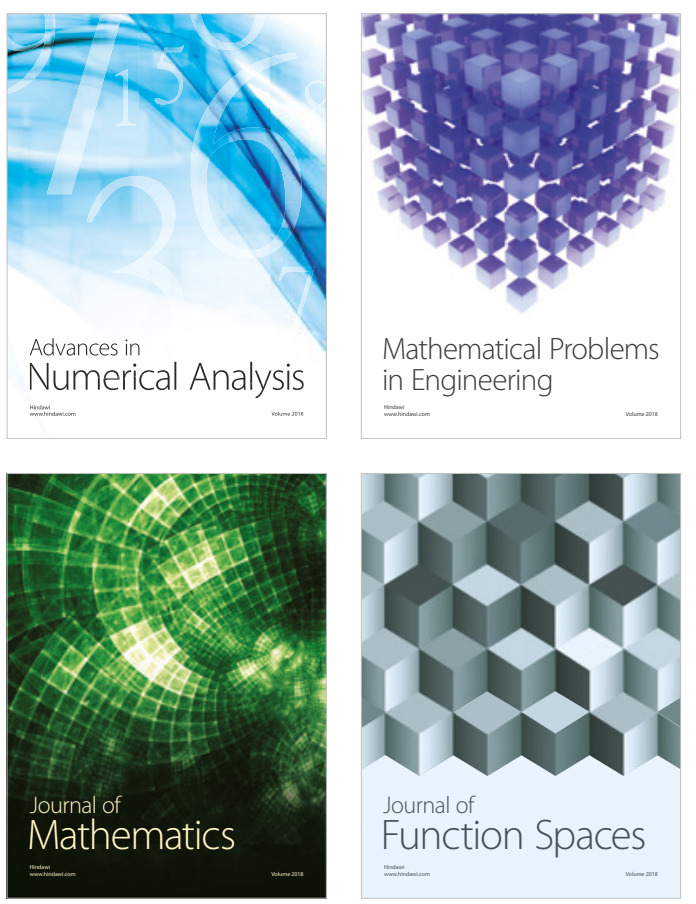

Mathematical Problems in Engineering

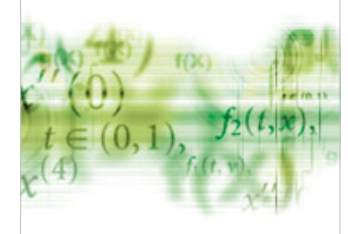

International Journal of

Differential Equations

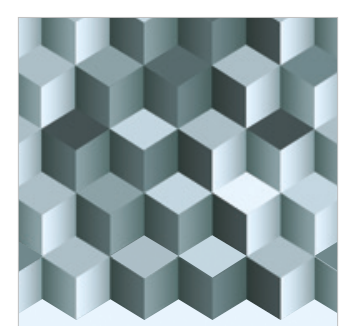

Journal of

Function Spaces

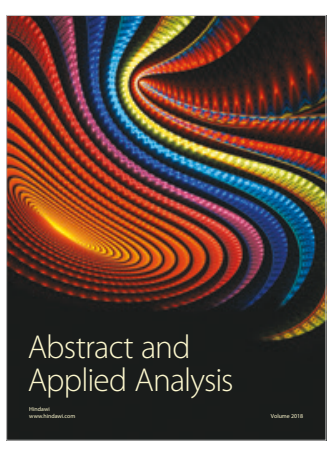

The Scientific

World Journal

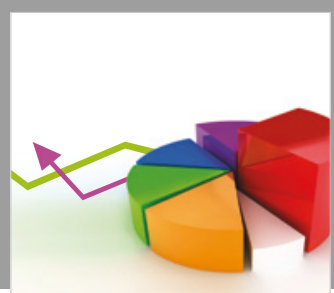

Journal of

Probability and Statistics
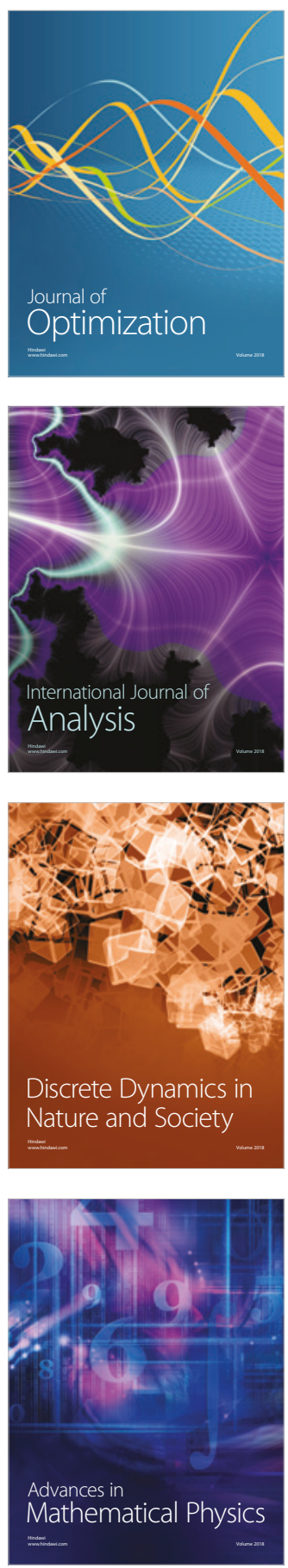